\title{
Combined salvage therapies for recurrent glioblastoma multiforme: treatment options in multifocal and multicentric patterns of recurrence
}

\begin{abstract}
Salvage therapies in recurrent multifocal glioblastoma

Background: Recurrent glioblastoma multiforme following multimodality therapy have limited palliative treatment options when the major modalities of therapy have been exhausted. Moreover, multifocal and multicentric bilateral patterns of recurrence is associated with worse prognosis and currently there is no specific treatment protocol for this patient population. The authors introduce a clinical and radiological indicationsolving algorithm and provide outcome rates of a multifocal and multicentric glioblastoma recurrence cohort.
\end{abstract}

Methods: Consecutive adult patients with multifocal and/or multicentric recurrence of glioblastoma who underwent a combined scheme of salvage treatments consisting of high dose rate (HDR) brachytherapy, stereotactic radiosurgery and chemotherapy were included in this retrospective study and were compared to a historical control group of 15 patients who have been treated with intensive temozolomide chemotherapy as the only treatment modality. All patients were previously treated with surgery of the primary pathology, concomitant and adjuvant radiochemotherapy with temozolomide.

Results: Median survival was 6months for the entire cohort after salvage treatment and can be translated into a 3month improvement in survival compared to the control group of patients with glioblastoma recurrence treated with temozolomide alone $(\mathrm{P}=0.033)$. HDR-Brachytherapy combined with stereotactic radiosurgery and chemotherapy renders acceptable complication rates and seems to be a reasonable salvage option for patients with multifocal and/or multicentric recurrent glioblastoma.

Conclusion: Our experience suggests that a combined salvage treatment plan appears to be either feasible or effective and can be considered in selected patients affected by multifocal and multicentric recurrence of glioblastoma. The authors' clinical and radiological indication-solving algorithm may assist in providing the best possible salvage treatment for this difficult population.

Keywords: glioblastoma multiforme, recurrence, multifocal, HDR brachytherapy, salvage therapy
Volume 7 Issue I - 2017

\author{
Eleftherios Archavlis \\ Department of Neurosurgery, University Medical Center, \\ Johannes Gutenberg-University Mainz, Germany
}

\begin{abstract}
Correspondence: Eleftherios Archavlis, Department of Neurosurgery, University Medical Centre, Johannes GutenbergUniversity Mainz, Langenbeckstrasse. I, D 55I3। Mainz, Germany, Tel 0049-|789|60743, Fax 0049-6|3|-17-2274, Email neurosurgery@t-online.de
\end{abstract}

Received: October 27, 2016 | Published: January 09, 2017
Abbreviations: CTCAE, common terminology criteria for adverse events; ddTMZ, dense dose Temozolomide; FSRS, fractionated stereotactic radiosurgery; HDR, high dose rat; KPS, Karnofsky performance status

\section{Statistical analysis}

\section{Background}

Despite the advancement made in the fields of neurosurgery, neuro-oncology, medical oncology, and radiation oncology, patients with recurrent high grade gliomas represent a unique challenge to the treating physicians. ${ }^{1,2}$ Gliomas have the ability to disseminate along established CNS routes, such as white matter tracts, cerebrospinal fluid, or local invasion. ${ }^{3}$ Multifocal appearance according to Budka et al. consists of tumors separated by white matter tracts within the same hemisphere, whereas multicentric glioma consists of tumors in opposite hemispheres or separated by the tentorium. ${ }^{4}$ Tumor dissemination can serve as a prognostic marker at the time of the primary diagnosis ${ }^{5}$ but a multifocal and multicentric recurrence of the glioblastoma into the surrounding and distant brain tissue is also associated with rapid clinical decline.
A multimodal and interdisciplinary approach to a patient with a recurrent high grade glioma involves various modalities, ranging from non-invasive techniques with three-dimensional (3-D) conformal external RT, ${ }^{6}$ intensity modulated radiotherapy (IMRT), ${ }^{7}$ stereotactic radiosurgery (SRS), ${ }^{8,9}$ stereotactic radiotherapy $(\mathrm{SRT})^{10}$ or systemic use of Temozolomide as dense dose treatment modality to invasive techniques such as re-operation (with or without chemotherapy with local therapy using Gliadel wafers) and brachytherapy (interstitial or intra-cavitary). ${ }^{10-15}$ But, for multifocal, multicentric recurrent glioblastomas, which are not amenable for reoperation there is no specific treatment protocol and unfortunately, these patients are often excluded from clinical trials.

Dose escalation with interstitial high dose rate (HDR) brachytherapy delivered with an automatic computer controlled afterloading device of a single 192Ir source combined with stereotactic radiosurgery and consecutive intensive temozolomide chemotherapy might prolong survival in patients with this devastating disease. In three retrospective studies we have reported the safety and feasibility of HDR-Brachytherapy and a survival benefit in comparison to conservative treatment for patients with recurrent glioblastoma. ${ }^{2,16,17}$ 
We designed a prospective study in order to report a treatment algorithm and outcome of combined therapeutic modalities used in a severely affected subgroup of patients with recurrent high grade gliomas, namely these with multifocal and /or multicentric patterns of recurrence.

\section{Methods}

Between 2008 and 2011, 66 consecutive adult patients with recurrent high grade gliomas were retrospectively analyzed. The validation cohort consisted of patients who underwent a multimodal treatment according to a clinical and radiological algorithm decided prior to the beginning of the series at Sana Klinikum Offenbach, Germany. We identified 10 patients with multifocal and 5 patients with a multicentric bihemispheric pattern of recurrence. We employed a historical control group of patients who were treated in the outpatient institute of Sana Klinikum Offenbach between 2006 and 2008, prior to the introduction of the above mentioned algorithm. Eligibility criteria included 1) histology proven diagnosis of glioblastoma (WHO grade $4)$, 2) age $\geq 18$ years, 3 ) primary treatment with initial surgery and radiotherapy with concomitant temozolomide, 4) patients with good neurological function (KPS >70), 5) multifocal and/or multicentric recurrence pattern and 6) provided informed consent for salvage therapy after recurrence. The exclusion criteria were the use of any other investigational agent, poor neurologic function $(\mathrm{KPS}<70)$ and conscious impairment.

All patients were treated for recurrence, having following possible combinations of treatment (treatment variables): a) HDRBrachytherapy followed by temozolomide and b) HDR-Brachytherapy followed by stereotactic radiosurgery and temozolomide. These different treatment groups were compared to historical patient group which was treated with intensive temozolomide chemotherapy as the only treatment modality. The clinical and radiological indicationsolving algorithm illustrated in Figure 1 was utilized to determine management decisions and was decided prior to the beginning of this series. Follow-up and follow-up MRI scans were performed at 3-month intervals until death. Brachytherapy catheters were placed percutaneously with CT-guidance. The number, depth and orientation of the catheters were selected so as to conform to the treatment planning volume after tumor demarcation with CT-based MR fused planning. HDR-Brachytherapy patients received 5.0 Gy twice a day with an interval of at least $6 \mathrm{~h}$ to a total dose of $40 \mathrm{~Gy}$. Patients with multicentric bihemispheric recurrence received salvage extended field stereotactic radiosurgery at a dose of $20 \mathrm{~Gy}$, extending the treatment margin by $0,5-1 \mathrm{~cm}$ beyond the enhancing region.

HDR-Brachytherapy and stereotactic radiosurgery were followed by temozolomide (TMZ) chemotherapy as an adjunctive treatment on a1week on $/ 1$ week off basis with a daily dose of $150 \mathrm{mg} / \mathrm{m}^{2}$, until documented disease progression or unacceptable toxicity. We chose this more rigorous dose regimen of TMZ according to the publications of Wick et al. who reported a 3months PFS as high as $48 \%$ with an overall survival for 12 months of $81 \% .^{18}$ Toxicity was evaluated according to the Common Terminology Criteria for Adverse Events (CTCAE; version 3.0). In case of hematological toxicity grade 4 or non hematological toxicity grade 3 , the dosage TMZ of the next cycle was reduced by $25 \mathrm{mg} / \mathrm{m}^{2} /$ day. In case of CTCAE grade 4 non hematological toxicity, the patient stopped treatment. In case of a grade 4 hematological toxicity or a grade 3 non-hematological toxicity at dose level of $75 \mathrm{mg} / \mathrm{m}^{2} /$ day, the patient went off treatment. In case of dose reductions, dose re-escalation was not allowed. Blood examinations were done on day 15 and day 29 and when platelets were above $100 \times 10^{9} / 1$ and neutrophils counts above $1.5 \times 109 / 1$, the following chemotherapy cycle was administered. Otherwise treatment was postponed until recovery to CTCAE grade $1 \mathrm{and} /$ or platelets were above $100 \times 10^{9} / 1$. The treatment was stopped if it had to be postponed for more than 2 cycles.

The primary endpoint of this study was disease-specific survival after salvage treatment. Secondary endpoints were complication rates, adverse events, mortality and morbidity due to toxicity. Progression was evaluated according to Macdonald criteria defined as " $>25 \%$ increase in size of enhancing tumor or any new tumor on CT or MR imaging, or neurologically worsening (that is, increase in the NIH-SS score $\geq 1$ compared to preceding visit) in case of stable or increased use of steroids". ${ }^{28}$ All patients were observed until the time of death or last follow-up. Median follow-up was 32months (range 28-36months). Karnofsky performance score (KPS) was estimated in both groups as an important parameter of quality of life before and after initiation of salvage treatment, as well as every three months during treatment.

All statistical analyses were conducted with BiAS software program (2006, Frankfurt, Germany). Mann-Whitney U test was applied as non-parametric significance test. In addition, Chi-quadrate $\left(\mathrm{Chi}^{2}\right)$ test and Fisher's exact test were performed to analyze the difference in patient characteristics, surgical parameters and complications for each surgical approach. P-values less than 0.05 were regarded as statistically significant. Kaplan-Meier survival analysis was obtained using long-rank tests.

\section{Results}

During the prospective period a total of 15 consecutive patients with recurrent multifocal and /or multicentral glioblastomas after initial surgery, radiotherapy and chemotherapy, underwent combined salvage treatment and were compared with a historical control group of 15 patients who underwent sole dens dose temozolomide chemotherapy. Table 1 summarizes the patients' demographics and tumor characteristics. The median patient age of the cohort was 57 years (range, 35 -76years). The mean KPS was 90 (range, 80-100). The primary tumor site was in all cases supratentorial. All study subjects had received external radiotherapy to whole brain and there was no possibility of further external re-irradiation. The prior doses of RT to whole brain were $40 \mathrm{~Gy}$. All patients were treated with systemic chemotherapy.

Out of the 15 patients with recurrent glioblastoma, 10 had a multifocal pattern of recurrence and underwent HDR Brachytherapy and 5 had a bihemispheric multicentric recurrence and underwent a combination of HDR Brachytherapy and stereotactic radiosurgery of the remote relapse regions. After completion of the local reirradiation, patients entered the systemic salvage chemotherapy. The salvage therapy data are summarized in Table 2. The median survival was 6 months for the combined salvage treatment cohort compared to 3 months in the sole ddTMZ group. The additional three-month improvement in medial survival time in patients with recurrent high grade gliomas represented a statistically significant increase compared to the sole ddTMZ group $(\mathrm{P}=0.043)$. Figure 2 graphically demonstrates this increase in survival for the patients with recurrent glioblastoma by 3 months for the group of combined salvage treatment compared to ddTMZ group.

The median KPS of the entire patient population was 80 at the time of salvage treatment. At the first month the median KPS was 80 for the combined salvage treatment group and 70 for the sole chemotherapy control group, respectively. Therefore, there was 
no severe deterioration regarding function immediately following salvage treatment in any of the groups.

Table 3 summarizes the side effects of the different combined salvage treatment as graded by the CTCAE criteria. There were no serious adverse HDR-Brachytherapy related effects and no grades 3 or 4 acute or late toxicities. Acute headache, vomiting and nausea (grade 1-2) was reported in 3/15 of patients which resolved after increase steroid administration. One intracerebral bleeding that occurred in the patient group undergoing combined salvage treatments was treated conservatively and had no neurologic sequel for the patient. Only 2 patients developed a symptomatic radiation necrosis identified on the follow-up MRI imaging. 3 patients in the ddTMZ control group developed adverse events and all of them discontinued TMZ due to toxicity: persistent grade 3 and 4 leucopenia (2) and grade 4 elevated transaminases (1). Two patients in the combined salvage treatment group developed toxicity in form of persistent grade 4 leucopenia and discontinued TMZ.

Table I Salvage therapy data of patients with recurrent multifocal/multicentric GBM

\begin{tabular}{|c|c|c|c|}
\hline Parameters & & $\begin{array}{l}\text { Combined Salvage } \\
\text { Treatment Group }\end{array}$ & ddTMZ \\
\hline no. of cases & & 15 & 15 \\
\hline Age & in years & 58,1 & 56,7 \\
\hline Sex (female), n (\%) & no. of cases & $6(40 \%)$ & $8(53 \%)$ \\
\hline KPS score & & 90 & 90 \\
\hline 100 & no. of cases & $4(27 \%)$ & $5(33 \%)$ \\
\hline 90 & no. of cases & $6(40 \%)$ & $8(53 \%)$ \\
\hline 80 & no. of cases & $5(33 \%)$ & $2(14 \%)$ \\
\hline $\begin{array}{l}\text { Time since initial } \\
\text { diagnosis }\end{array}$ & in months & 9,4 & 9,1 \\
\hline $\begin{array}{l}\text { Side of tumor } \\
\text { location }\end{array}$ & no. of cases & & \\
\hline Left & & $9(60 \%)$ & $8(53 \%)$ \\
\hline Right & & $6(40 \%)$ & $7(47 \%)$ \\
\hline $\begin{array}{l}\text { Predominant lobe } \\
\text { of tumor location }\end{array}$ & no. of cases & & \\
\hline Frontal & & $6(40 \%)$ & $4(27 \%)$ \\
\hline Temporal & & $4(27 \%)$ & $5(33 \%)$ \\
\hline Parietal & & $3(20 \%)$ & $3(20 \%)$ \\
\hline Occcipital & & $2(13 \%)$ & $3(20 \%)$ \\
\hline $\begin{array}{l}\text { Eloquent/critical } \\
\text { regions involved }\end{array}$ & no. of cases & & \\
\hline 0 & & $5(33 \%)$ & $5(33 \%)$ \\
\hline I & & $5(33 \%)$ & $6(40 \%)$ \\
\hline 2 & & $3(20 \%)$ & $3(20 \%)$ \\
\hline 3 & & $2(14 \%)$ & I (6\%) \\
\hline Tumor volume & $\mathrm{cm}^{3}$ & 26,1 & 25,3 \\
\hline Seizures & no. of cases & & \\
\hline Yes & & $8(53 \%)$ & $10(67 \%)$ \\
\hline No & & 7 (47\%) & $5(33 \%)$ \\
\hline Midline shift & no. of cases & & \\
\hline Yes & & $8(53 \%)$ & $5(33 \%)$ \\
\hline No & & 7 (47\%) & $10(67 \%)$ \\
\hline Cerebral edema & no. of cases & & \\
\hline None & & I (6\%) & $2(14 \%)$ \\
\hline Perifocal $\leq 2 \mathrm{~cm}$ & & 7 (47\%) & $8(53 \%)$ \\
\hline Perifocal $\geq 2 \mathrm{~cm}$ & & $7(47 \%)$ & $5(33 \%)$ \\
\hline
\end{tabular}

Table 2 Salvage therapy data of patients with recurrent multifocal/multicentric GBM

\begin{tabular}{llll}
\hline Parameters & \multicolumn{2}{l}{$\begin{array}{l}\text { Combined Salvage Treatment } \\
\text { Group }\end{array}$} & ddTMZ \\
\hline Median Survival after Salvage Treatment & 6 months & 3 months \\
& Multifocal & 6,2 months & 3,3 \\
Overall Survival & Multicentric & 5,8 months & 2,7 \\
& Multifocal & 17 months & 14 months \\
& Multicentric & 18 & 14,2 \\
Progression Free Survival & 3 months & 13,8 \\
& Multifocal & 3,5 & 0 \\
& Multicentric & 2,5 & 0 \\
\hline
\end{tabular}

* Mean values are presented as the means

Table 3 Complication rates and adverse events (AEs) stratified by treatment group

\begin{tabular}{lll}
\hline Parameters & $\begin{array}{l}\text { Combined Salvage } \\
\text { Treatment Group }\end{array}$ & ddTMZ \\
\hline No. of cases & 15 & 15 \\
AEs due to toxicity & 2 & 3 \\
all & 0 & 8 \\
Grade 3/4* & & \\
Thrombocytopenia & 1 & $\mathrm{I}$ \\
Grade 3/4 & & \\
Leucopenia & $\mathrm{I}$ & $\mathrm{I}$ \\
Grade 3/4 & & $\mathrm{I}$ \\
Elevated transaminases & & 0 \\
Grade 3/4 & 0 & 0 \\
Intracerebral bleeding & $\mathrm{I}$ & 3 (20\%) \\
Radiation necrosis & 2 & \\
Overall Complications & $5(33 \%)$ & \\
\hline
\end{tabular}

*According to the Common Toxicity Criteria

\section{Discussion}

High grade gliomas tend to recur within 6-10months after treatment. The patterns of recurrence and failure studies examining recurrence of glioblastoma multiforme after primary therapy have demonstrated that majority of the patients experience recurrence immediately near the resection bed (within $2 \mathrm{~cm}$ ). ${ }^{13,19}$ Disease progression is followed shortly by death. The importance of complete surgical resection has been discussed in the literature..$^{20,21}$ Multifocal and multicentric patterns of recurrence have been associated with a worse prognosis than solitary recurrence. Local therapies in order to offer local control have disappointing results, resurgery poses several technical challenges, including bigger tumor volumes, significant gliosis and adhesions, and proximity of the tumor to eloquent brain $\operatorname{areas}^{22,23}$ and is likely to result in complications and injury to the normal brain tissue. On the other hand, dose escalation with external beam radiotherapy in preirradiated areas did not result in benefit of improved survival and can lead to radionecrosis. ${ }^{24}$

A number of attempts have been reported to escalate the dose with interstitial brachytherapy while limiting doses received by the healthy brain tissue. ${ }^{10,25,26}$ The addition of stereotactic radiosurgery in 
combination to chemotherapy could have beneficial effects. Previous studies show the effectiveness of fractionated stereotactic radiosurgery (FSRS) as an option for the treatment of recurrent glioblastoma. ${ }^{27,28}$ Combs et al. reported 5 months progression-free survival after FSRT and 21 months median overall survival. ${ }^{29}$ Koga et al., ${ }^{8}$ recently demonstrated 24 months median overall survival in patients receiving salvage gamma knife radiosurgery at a dose of $20 \mathrm{~Gy} .{ }^{8}$ However, SRS is not recommended for recurrent lesions $>40 \mathrm{~mm}$ in diameter and the fulfilment of this restriction immensely limits patient's eligibility for treatment. ${ }^{30}$

The HDR-Brachytherapy delivered through catheters with an automatic computer controlled afterloading device of a single 192Ir source as practiced in our hands, is an attractive form of localized radiation treatment that involves delivery of highly localized doses of radiation implanted not only in solid and cystic parts of the tumor but also in the former resection cavity while minimizing dose deposition to the surrounding tissues. Further, this method allows the delivery of radiation dose to areas most at risk of recurrence while addressing the limitations of interstitial (seed) and intracavitary brachytherapy (Gliasite). HDR-Brachytherapy offers radiobiological advantages in comparison to radiosurgery in the treatment of larger tumor recurrence.

In contrast to brachytherapy, the data on chemotherapy use in the setting of a high gradegliomas recurrence are plentiful. Prior to the advent of TMZ era, there was considerable debate about whether chemotherapy was useful in the treatment of malignant gliomas at all. A landmark phase 3 study published in 2005 established TMZ as the current standard of care. ${ }^{31}$ In this study, patients were randomized to either postresection RT alone or postresection RT with TMZ. The RT with concurrent TMZ group demonstrated a statistically significant improved median survival of 14.6 versus 12.1 months as well as improved 2-year survival rates of $26.5 \%$ versus $10.4 \%$. In the 5 -year follow-up analysis of the trial, the benefit of TMZ was confirmed in all clinical prognostic subgroups receiving the drug. ${ }^{32}$ Overall survival at 5years was $9.8 \%$ in the RT plus TMZ group compared with $1.9 \%$ in the radiotherapy alone group. Temozolomide is usually well tolerated with myelosuppression as the most commonly reported toxicity. Grade III or IV events occurred in around $15 \%$ of patients receiving TMZ. In one of the largest series reported to date, temozolomide alone as applied for a recurrent glioblastoma resulted in a 1-month median survival. ${ }^{14}$ Other studies on different new chemotherapeutic agents report a range of survival, from six to 26 months. ${ }^{18,24,33-37}$

Many Phase II trials, conducted before the era of combined RT/ TMZ for newly diagnosed GBM, reported PFS-6 rates of up to $39 \%$ in patients with recurrent disease receiving TMZ in combination with other agents (i.e., marimastat, ${ }^{38}$ cisplatin $^{39}$ and 13 -cis-retinoic acid. ${ }^{40}$ However, it has not yet been proven that multiagent chemotherapy is more effective than single nitrosourea administration. ${ }^{41}$ Nor has it been demonstrated that TMZ has advantages over a BCNU or procarbazine, lomustine and vincristine regimen, despite the fact that an indirect comparison made between studies suggests that TMZ has a better, noncumulative toxicity profile than other regimens used.

Following the introduction of the current standard of care for newly diagnosed GBM patients with RT and concomitant/adjuvant TMZ, novel first- and second-line treatments are under evaluation. Therefore, even though clear data are not yet available, a TMZ rechallenge for patients without disease progression during TMZ treatment should be considered a reasonable option..$^{42}$ Temozolomide rechallenge was recently investigated also by Perry et al., ${ }^{37}$ In their retrospective analysis, the authors showed that TMZ rechallenge with a continuous $50 \mathrm{mg} / \mathrm{m} 2$ daily schedule is an intriguing approach, especially for patients with recurrence after completion of TMZ administration concurrent with and adjuvant to RT.Currently, there are no clear guidelines regarding the optimal management of a multifocal and/or multicentric glioblastoma recurrence and salvage therapy is based on surgeon's preference and varies among centers. To the best of our knowledge, there are no reports available based on an algorithm of combined treatments including interstitial brachytherapy, stereotactic radiosurgery and temozolomide chemotherapy. In the present study, 15 patients received salvage treatment according to the indication solving algorithm depicted in Figure 1.

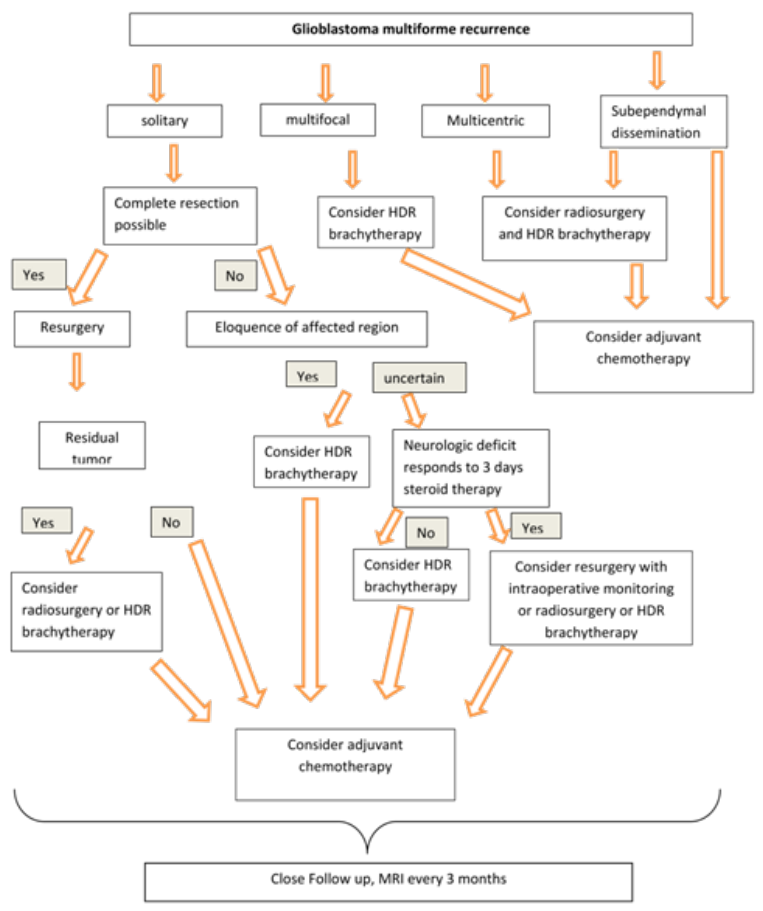

Figure I Flow chart that shows the different therapeutic modalities of the multimodal treatment group depending on the pattern of recurrence.

There were 5 complications, yielding an 33\% complication rate in this challenging patient population; in comparison the sole ddTMZ group presented a $20 \%$ complication rate due to toxicity. Our present study showed that combined therapies could achieve better long-term survival outcomes to those seen with sole temozolomide chemotherapy in patients with recurrent multifocal and /or multicentric glioblastoma. It is obvious that a non randomized comparison of combined salvage treatments with ddTMZ on which the above conclusions are drawn is complicated by bias of patient selection. Moreover, it was not the aim of this study to determine the most appropriate first-line chemotherapy at first recurrence. We chose TMZ for our patients with recurrent glioblastoma because it is well tolerated, has good oral bioavailability, and is convenient to administer as an outpatient regimen. Additionally, there is no clear benefit when comparing PCV with TMZ. ${ }^{43}$ Bevacizumab has been adopted into regular clinical practice, though there is no phase III evidence of its efficacy and based on the current literature there is a modest response rate and effect on progression-free survival. In the absence of impressive clinical trial data which support bevacizumab and considering its complication rates we decided not to use bevacizumab in a regular clinical base. 


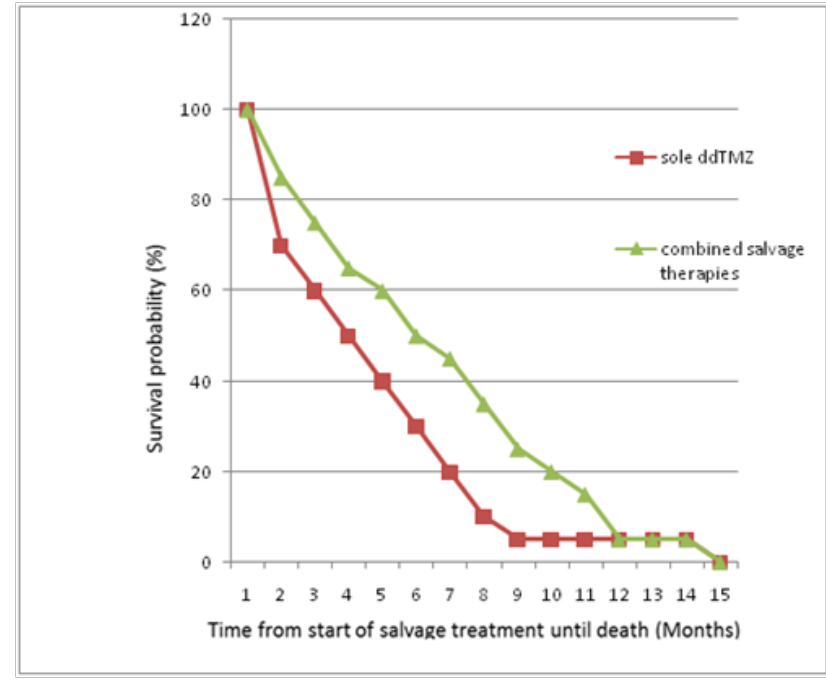

Figure 2 Kaplan-Meier plots of patients stratified according to therapeutic modality.

In our hands HDR-Brachytherapy is an approved treatment method of recurrent high grade gliomas. It offers a potential advantage over the conventional interstitial brachytherapy by means of delivering a more conformal, uniform and relatively homogeneous dose distribution, avoiding the "hot" and "cold" spots of over- and under-dosing of seed or strand brachytherapy approaches. ${ }^{27}$ Furthermore, the catheters and subsequent treatment planning conform to the surgical cavity, and the dosimetry is precise and easily reproduced. This treatment method eliminates the need for stereotactic head frame, multiple scalp incisions and bur holes in the operating room under general anesthesia. Thus, the catheters are placed in the CT scan and allow delivery of high radiation dose to the contrast enhancing areas with a margin of $1 \mathrm{~cm}$ when this is possible considering the presence of high risk eloquent regions while limiting the risk of radio necrosis by using multiple radiation delivery vehicles that produce a homogeneous dose distribution.

Our retrospective study indicates that catheter implantation, dose escalation with HDR-Brachytherapy and stereotactic radiosurgery in combination with chemotherapy are feasible, safe and well-tolerated by most of the patients. The encouraging survival and local control results indicate that the approach with combined salvage treatment may provide benefit to patients with recurrent high grade gliomas. In this trial, combined salvage treatments translated to an average mean overall survival time of 16 months and a median survival after salvage treatment of 6months. The additional three-month improvement in overall und post-salvage treatment survival time, observed in this cohort of multifocal/multicentric recurrent glioblastoma, was corroborated as a statistically significant increase as compared to the control group of chemotherapy alone. The small number of radiation necrosis in this study cohort suggests that patients may tolerate this regimen better than the one with interstitial seed brachytherapy.

None of our patients required surgical intervention to address radiation necrosis. Same results regarding radiation necrosis reported Wernicke at al. ${ }^{15}$ with a regimen of GliaSite brachytherapy. ${ }^{15}$ HDRBrachytherapy and stereotactic radiosurgery offer a possibility of further dose escalation with the potential to improve the efficacy of this treatment while avoiding the morbidity associated with high rates of radiation necrosis and implantation-explantation surgery. The rate of the acute and long-term toxicities was acceptable. This report gives a full account of patients with multifocal and /or multicentral recurrent glioblastoma treated HDR-Brachytherapy and stereotactic radiosurgery combined with dense dose chemotherapy and examines survival and complication rates, as well as compares and contrasts the outcomes and toxicity rates to a control group of patients treated with ddTMZ alone. We developed a treatment algorithm guiding the neurotherapist through the decision making process. The present results might therefore help neurotherapists to put different treatment modalities into perspective.

Of note, patients in this study were retrospectively evaluated and not randomly assigned to therapeutic modality which warrants cautious interpretation due to possible selection bias. Another drawback of the study that undermines reliability is the small number of patients included. Large number of patients could be adopted in a multicenter study which we are planning in the near future. ${ }^{44-48}$

\section{Conclusion}

The objective of this prospective study was to assess survival rates of combined salvage treatments after multifocal and/or multicentric patterns of recurrence of glioblastoma and to evaluate side effects. This study demonstrates that HDR-Brachytherapy in combination with stereotactic radiosurgery and chemotherapy can be valuable tools for therapeutic escalation in this subgroup of patients. We propose the aforementioned clinical and radiological algorithm to systematically assist therapists in their efforts to provide the best possible salvage treatment in this difficult patient population.

Header for declaration: Salvage therapies in recurrent multifocal glioblastoma Ethics approval and consent to participate: Ethics approval was not needed as this was a retrospective study and all investigations and therapies performed were part of standardized routine.

\section{Availability of data and materials}

Authors can make freely available to colleagues in academic research upon request any form of published data for appropriate scientific use.

\section{Acknowledgments}

None.

\section{Competing Interest}

The authors report no conflict of interest concerning the materials and methods used in this study or the findings specified in this paper.

\section{References}

1. Affronti ML, Heery CR, Herndon JE, et al. Overall survival of newly diagnosed glioblastoma patients receiving carmustine wafers followed by radiation and concurrent temozolomide plus rotational multiagent chemotherapy. Cancer. 2009;115(15):3501-3511.

2. Archavlis E, Tselis N, Birn G, et al. Survival analysis of HDR brachytherapy versus reoperation versus temozolomide alone: a retrospective cohort analysis of recurrent glioblastoma multiforme. $B M J$ Open. 2013;3(3).

3. Barker FG, Chang SM, Gutin PH, et al. Survival and functional status after resection of recurrent glioblastoma multiforme. Neurosurgery. 1998;42(4):709-720.

4. Budka H, Podreka I, Reisner T, et al. Diagnostic and pathomorphological aspects of glioma multiplicity. Neurosurg Rev. 1980;3(4):233-241. 
5. Parsa AT, Wachhorst S, Lamborn KR, et al. Prognostic significance of intracranial dissemination of glioblastoma multiforme in adults. $J$ Neurosurg. 2005;102(4):622-628.

6. Kim HK, Thornton AF, Greenberg HS, et al. Results ofre-irradiation of primary intracranial neoplasms with three-dimensional conformal therapy. Am J Clin Oncol. 1997;20(4):358-363.

7. Fuller CD, Choi M, Forthuber B, et al. Standard fractionation intensity modulated radiation therapy (IMRT) of primary and recurrent glioblastoma multiforme. Radiat Oncol. 2007;14(2):26.

8. Shaw E, Scott C, Souhami L, et al. Radiosurgery for the treatment of previously irradiated recurrent primary brain tumors and brain metastases: initial report of radiation therapy oncology group protocol. Int J Radiat Oncol Biol Phys. 1996;34(3):647-654

9. Villavicencio AT, Burneikiene S, Romanelli P, et al. Survival following stereotactic radiosurgery for newly diagnosed and recurrent glioblastoma multiforme: a multicenter experience. Neurosurg Rev. 2009;32(4):417-424.

10. Leibel SA, Gutin PH, Wara WM, et al. Survival and quality of life after interstitial implantation of removable high-activity iodine-125 sources for the treatment of patients with recurrent malignant gliomas. Int $J$ Radiat Oncol Biol Phys. 1989;17(6):1129-1139.

11. Brem H, Piantadosi S, Burger PC, et al. Placebo-controlled trial of safety and efficacy of intraoperative controlled delivery by biodegradable polymers of chemotherapy for recurrent gliomas. The Polymer-brain Tumor Treatment Group. Lancet. 1995;345(8956):1008-1012.

12. Park JK, Hodges $\mathrm{T}$, Arko L, et al. Scale to predict survival after surgery for recurrent glioblastoma multiforme. $J$ Clin Oncol. 2010;28(24):3838-3843.

13. Chan JL, Lee SW, Fraass BA, et al. Survival and failure patterns of highgrade gliomas after three-dimensional conformal radiotherapy. J Clin Oncol. 2002;20(6):1635-1642.

14. Trent S, Kong A, Short SC, et al. Temozolomide as second-line chemotherapy for relapsed gliomas. J Neurooncol. 2002;57(3):247-251.

15. Wernicke AG, Sherr DL, Schwartz TH, et al. The role of dose escalation with intracavitary brachytherapy in the treatment of localized CNS malignancies: outcomes and toxicities of a prospective study. Brachytherapy. 2010;9(1):91-99.

16. Tselis N, Kolotas C, Birn G, et al. CT-guided interstitial HDR brachytherapy for recurrent glioblastoma multiforme. Long-term results. Strahlenther Onkol. 2007;183(10):563-570.

17. Kolotas C, Birn G, Baltas D, et al. CT guided interstitial high dose rate brachytherapy for recurrent malignant gliomas. $\mathrm{Br} J$ Radiol. 1999;72(860):805-808.

18. Wick W, Steinbach JP, Küker WM, et al. One week on/one week off: a novel active regimen of temozolomide for recurrent glioblastoma. Neurology. 2004;62:2113-2115.

19. Aydin H, Sillenberg I, von Lieven H. Patterns of failure following CT-based 3-D irradiation for malignant glioma. Strahlenther Onkol. 2001;177(8):424-431.

20. Stummer W, Pichlmeier U, Meinel T, et al. Fluorescence-guided surgery with 5-aminolevulinic acid for resection of malignant glioma: a randomised controlled multicentre phase III trial. Lancet Oncol. 2006;7(5):392-401

21. Stummer W, Reulen HJ, Meinel T, et al. Extent of resection and surviva in glioblastoma multiforme: identification of and adjustment for bias. Neurosurgery. 2008;62(3):564-576.

22. Batzdorf U, Malamud N. The problem of multicentric gliomas. $J$ Neurosurg. 1963;20:122-136.
23. Dirks P, Bernstein M, Muller PJ, et al. The value of reoperation for recurrent glioblastoma. Can J Surg. 1993;36(3):271-275.

24. Gerstein J, Franz K, Steinbach JP, et al. Postoperative radiotherapy and concomitant temozolomide for elderly patients with glioblastoma. Radiother Oncol. 2010;97:382-386.

25. Bernstein M, Laperriere N, Glen J, et al. Brachytherapy for recurrent malignant astrocytoma. Int J Radiat Oncol Biol Phys. 1994; 30(5): 1213-1237.

26. Scharfen CO, Sneed PK, Wara WM, et al. High activity iodine-125 interstitial implant for gliomas. Int $J$ Radiat Oncol Biol Phys. 1992;24(4):583-591.

27. Barbarisi M, Romanelli P. The emerging role of stereotactic radiosurgery in the treatment of glioblastoma multiforme. Curr Radiopharm. 2012;5(4):292-293.

28. Fokas E, Wacker U, Gross MW, et al. Hypofractionated stereotactic reirradiation of recurrent glioblastomas: a beneficial treatment option after high-dose radiotherapy? Strahlenther Onkol. 2009;185(4):235-240.

29. Combs SE, Thilmann C, Edler L, et al. Efficacy of fractionated stereotactic reirradiation in recurrent gliomas: long-term results in 172 patients treated in a single institution. J Clin Oncol. 2005;23:8863-8869.

30. Shaw E, Scott C, Souhami L, et al. Radiosurgery for the treatment of previously irradiated recurrent primary brain tumors and brain metastases: initial report of radiation therapy oncology group protocol. Int J Radiat Oncol Biol Phys. 1996;34(3):647-654.

31. Stupp R, Mason WP, van den Bent MJ, et al. Radiotherapy plus concomitant and adjuvant temozolomide for glioblastoma. $N$ Engl $J$ Med. 2005;352(10):987-996.

32. Stupp R, Hegi ME, Mason WP, et al. European Organisation for Research and Treatment of Cancer Brain Tumour and Radiation Oncology Groups; National Cancer Institute of Canada Clinical Trials Group. Effects of radiotherapy with concomitant and adjuvant temozolomide versus radiotherapy alone on survival in glioblastoma in a randomised phase III study: 5-year analysis of the EORTC-NCIC trial. Lancet Oncol. 2009;10(5):459-466.

33. Taal W, Segers-van Rijn JM, Kros JM, et al. Dose dense 1 week on/1 week off temozolomide in recurrent glioma: a retrospective study. $J$ Neurooncol. 2012;108(1):195-200.

34. Gállego Pérez-Larraya J, Lahutte $M$, et al. Response assessment in recurrent glioblastoma treated with irinotecan-bevacizumab: comparative analysis of the Macdonald, RECIST, RANO, and RECIST + F criteria. Neuro Oncol. 2012;14:667-73.

35. Friedman HS, Prados MD, Wen PY, et al. Bevacizumab alone and in combination with irinotecan in recurrent glioblastoma. J Clin Oncol. 2009;27(28):4733-4740.

36. Kreisl TN, Kim L, Moore K, et al. Phase II trial of single-agent bevacizumab followed by bevacizumab plus irinotecan at tumor progression in recurrent glioblastoma. JClin Oncol. 2009;27(5):740-745.

37. Perry JR, Bélanger K, Mason WP, et al. Phase II trial of continuous doseintense temozolomide in recurrent malignant glioma: RESCUE study. $J$ Clin Oncol. 2010;28(12):2051-2057.

38. Adamson C, Kanu OO, Mehta AI, et al. Glioblastoma multiforme: a review of where we have been and where we are going. Expert Opin Investig Drugs. 2009;18(8):1061-1083.

39. Li J, Wang M, Won M, et al. Validation and simplification of the Radiation Therapy Oncology Group recursive partitioning analysis classification for glioblastoma. Int J Radiat Oncol Biol Phys. 2011;81(3):623-630.

40. Paravati AJ, Heron DE, Landsittel D, et al. Radiotherapy and temozolomide for newly diagnosed glioblastoma and anaplastic astrocytoma: validation of Radiation Therapy Oncology GroupRecursive Partitioning Analysis in the IMRT and temozolomide era. $J$ Neurooncol. 2011;104(1):339-349. 
41. Siker ML, Wang M, Porter K, et al. Age as an independent prognostic factor in patients with glioblastoma: a Radiation Therapy Oncology Group and American College of Surgeons National Cancer Data Base comparison. J Neurooncol. 2011;104(1):351-356.

42. Park $\mathrm{CK}$, Lee $\mathrm{SH}$, Han $\mathrm{JH}$, et al. Recursive partitioning analysis of prognostic factors in WHO grade III glioma patients treated with radiotherapy or radiotherapy plus chemotherapy. BMC Cancer. 2009;9:450

43. Brada M, Stenning S, Gabe R, et al. Temozolomide versus procarbazine, lomustine, and vincristine in recurrent high-grade glioma. J Clin Oncol. 2010;28(30):4601-4618.

44. Quant EC, Drappatz J, Wen PY, et al. Recurrent high-grade glioma. Curr Treat Options Neurol. 2010;12(4):321-333.
45. Macdonald DR, Cascino TL, Schold SC, et al. Response criteria for phase II studies of supratentorial malignant glioma. J Clin Oncol. 1990;8(7):1277-1280.

46. Koga T, Maruyama K, Tanaka M, et al. Extended field stereotactic radiosurgery for recurrent glioblastoma. Cancer. 2012;118(17):4193-4200.

47. Archavlis E, Tselis N, Birn G, et al. Combined salvage therapies for recurrent glioblastoma multiforme: evaluation of an interdisciplinary treatment algorithm. J Neurooncol. 2014;119(2):387-395.

48. Archavlis E, Tselis N, Birn G, et al. Salvage therapy for recurrent glioblastoma multiforme: a multimodal approach combining fluorescence-guided resurgery, interstitial irradiation, and chemotherapy. Neurol Res. 2014;36(12):1047-1055. 